\title{
Beam Loading Effect in SSCL Coupled Cavity Linac
}

\author{
Yu. Senichev, R. Cutler, J. Hurd, D. Raparia \\ Superconducting Super Collider Laboratory* \\ 2550 Beckleymeade Ave., Dallas, TX 75237
}

\section{INTRODUCTION}

The Superconducting Super Collider Accelerating Complex consists of a cascade of accelerator and each one of them accelerates the beam to some energy and then transfers it to the next stage. The design luminosity of $10^{33} \mathrm{~cm}^{-2} \mathrm{~s}^{-1}$ is obtained by the high efficiency of all stages and the ability to accelerate beam with as minimum emittance growth as possible. For this purpose the Linac pulse length is specified as $9 \mu \mathrm{s}$ in order to minimize the number of target crossings and so to minimize the emittance growth during charge exchange injection into the LEB booster.

However, the transient caused by switching on the beam makes up a significant part of the pulse length and can influence the characteristics of beam. In this paper we present the results of the transient study of the conpled cavity linac and discuss the model that predicts the parametric resonance between the beam and the field distortion settup during transient.

\section{RF TRANSIENT IN CAVITY}

CCL structure consists of 9 modules and each one of them has 8 tanks [1]. Each CCL tank is formed by brazing together 16 identical accelerating cells and 17 identical coupling cells with $7 \%$ coupling coefficient. The tanks are connected with bridge couplers that have $16 \%$ coupling coefficient. The power from the generator is input into the central bridge.

The excitation of the module can be described by a system of normal modes for the periodical chain of coupled cells (accelerating and coupling). Every cell is considered in a single mode approximation. We shall solve the problem of beam loading and compensation of beam's electromagnetic field perturbation by the generator. It is assumed that at the moment $t=0$ the beam enters the resonator(in our case module). Simultaneousiy, the generator gives the additional power required to compensate for beam loading. In common form, the total electromagnetic field is described by the equation:

$$
\begin{array}{r}
E(x, y, z, t)=\operatorname{Re}\left[\sum_{s}^{N} \frac{Q_{s}}{N_{s} \omega_{s}} a_{s}(t) e^{i h s z}\right. \\
\left.E_{0}(x, y, z)\left[\int_{V} j^{g} E_{s}^{*} d v-\int_{V} j^{e} E_{s}^{*} d v\right] e^{-i w t}\right],
\end{array}
$$

-Operated by the Universities Research Association, Inc., for the U.S. Department of Energy under Contract No. DE-AC3589ER40486. where

$$
\begin{array}{r}
a_{s}(t)=\frac{1-e^{-i \frac{\omega_{s}^{2}-\omega^{2}}{2 \omega} t} e^{-\frac{\omega^{2}}{2 \omega_{0}} t}}{1+i \frac{\omega_{t}^{2}-\omega^{2}}{\omega_{d}^{2}} Q_{s}} \\
\omega_{s}^{2}-\omega^{2}=K_{f} \cos \left(h_{s}\right) \\
N_{s}=\varepsilon \int_{V} E_{s} E_{s}^{*} d v
\end{array}
$$

where $j^{g}$ and $j^{e}$ are the generator and beam currents, $K_{f}$ is the coupling coefficient. $N$, is a norm, $Q$, is a quality factor, $h_{s}$ is a wave number and $\omega_{s}$ is a frequency of $s$-th mode. The first integral describes the additional power of the generator and the second one is the excitation by beam. The coefficients outside the integrals are the same, so the beam loading compensation in the trivial case is when the integrals cancel. However, in practice it is possible to compensate only for one mode since the character of the interaction of the beam and of the generator with the resonator is quit difference. Actually the beam and the generator currents can be written as

$$
\begin{gathered}
j^{e}(z, t)=j_{1}(z) e^{-i\left(\omega t-h_{b} z\right)} \\
j^{g}(z, t)=j_{1} \delta\left(z-z_{0}\right) e^{-i \omega t},
\end{gathered}
$$

where $h_{b}=\omega / v_{b}, v_{b}$ is beam velocity, $z_{0}$ is point of power input into the resonator, $j_{1}$ means first harmonic and the frequency of the beam and the generator equal each to other. Here the magnitude of the currents is taken as an average over the cross section of the beam and the loop (or hole) since we study the excitation of axial symmetrical modes only. It is obviously that the integral of the beam interaction for a constant first harmonic along the resonator doesn't equal to zero except for fundamental mode $\left(s=s_{f}\right)$ under the condition when $h_{s}=h_{v}$. At the same time the integral for generator doesn't equal zero for any mode if the coupling coefficient $\operatorname{Re}\left[e^{i h_{\mathrm{x}}=0}\right]$ has a "nonzero" magnitude. In other words, in order to compensate the beam perturbation of the fundamental mode, we have to input power which equals the beam power but simultaneously this local input of power excites all modes. Let's rewrite the expression (3) for the field induced by beam, taking into account that only the fundamental mode will be excited:

$$
E_{b 1}=R e\left[\left(1-e^{-\frac{\breve{s}}{2 Q_{0}} t}\right) e^{i h_{b} z} E_{0}(x, y, z) e^{-i\left(\omega t+\phi_{\star}\right)} R P\right],
$$

where $R P=\frac{P_{\text {beam }}}{P_{\text {Ios }} \cos \phi_{\text {s }}}$.

Here we have taken $P_{l o s}=\frac{N_{1}}{2 Q_{0}}, Q_{0}$ is the loaded quality factor of the resonator for the fundamental mode, 
$P_{\text {beam }}=\frac{1}{2} \cos \phi_{s} \int_{v} j_{1}^{e} E_{s}^{*} d v$, and $\phi_{s}$ is the synchronous phase of beam. Now since we are interested in the effect of the interaction of the beam with the perturbed field by itself, let's represent the field of the generator in the view of the travelling harmonics $E_{1}=0.5 E_{0}$ with the phase velocity equal the beam velocity:

$E(x, y, z, t)=\operatorname{Re}\left[\sum_{j}^{N} B_{s}(z, t) 2 E_{1} e^{-i\left(\omega t-h_{b} z+\phi_{s}\right)}\right] R P$

where

$$
\begin{aligned}
& B_{s}(z, t)=e^{-i\left[(\omega,-\omega) t-\left(h_{,}-h_{b}\right) z\right]} \\
& \mathbf{x}\left\{\begin{array}{cl}
\left(1-e^{-\frac{\omega}{2 Q_{0}} t}\right) & \text { if } s=s_{f} \\
-\frac{e^{-\frac{\omega_{t}^{2}}{2 \omega Q} t}}{1+i \frac{\omega_{z}^{2}-\omega^{2}}{\omega^{3}} Q}, & \text { otherwise }
\end{array}\right.
\end{aligned}
$$

From this expression one can see that the space-time distortion (front) of the electromagnetic field with the carrier resonant frequency moves along the resonator with a velocity equal to average velocity for all modes:

$$
v_{\text {front }}=\overline{\left(\omega_{s}-\omega\right) /\left(h_{s}-h_{b}\right)}
$$

Since

$$
\lim _{\omega_{s}-\omega \rightarrow 0}\left(\omega_{s}-\omega_{b}\right) /\left(h_{s}-h_{b}\right)=v_{\text {group }}
$$

then it means that the front of perturbation which the beam "sees" moves with the group velocity. It is obviously that each mode has its own group velocity and moreover, it damps slightly differently. Due to this the front will change shape as it moves along the resonator. $B_{s}(z, t)$ for $s=s_{f}$ is the average of the electromagnetic field over the

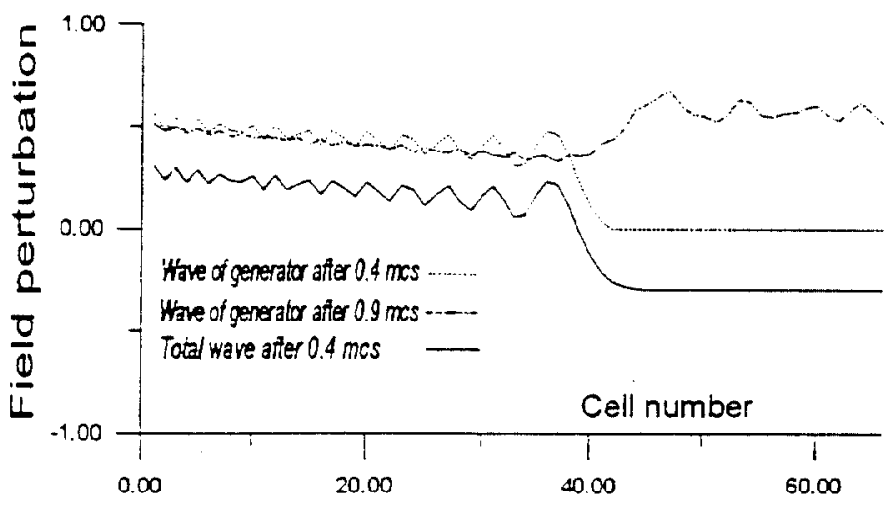

Figure 1. Field perturbation along module

length of the resonator at any moment in time. It is exactly equal to the field induced by beam, when $P_{\text {beam }}=P_{\text {gener }}$. So if this term subtracted from equation(9) then we shall get field which the beam sees. Knowing the dispersion function of the structure, it is possible to calculate what the distribution of electromagnetic field will be during the transient. Figure 1 shows the field of generator at the moments $t=0.4 \mu \mathrm{s}$ and $0.9 \mu \mathrm{s}$ and total field at the moment $t=0.4 \mu \mathrm{s}$. This picture explains what will happen in the cavity during injection of beam and simultaneous input of power. The origin of coordinates corresponds to the point of the power input. In the case of the SSC, this is the middle of the resonator. Physically, it means that the beam moves with the phase velocity and fills the resonator $1 / K_{f}$ as fast as the wave front from generator. So after first tens of $n s$ the beam interacts with the whole resonator and the average level of field will decrease in accordance with the exponential law $\sim\left(1-e^{-\omega t / 2 Q_{0}}\right)$. The generator creates a wave which radiates in both directions from the middle and divides the resonator into three parts with alternative drops of field. The overfall change in time goes as $\sim e^{-\omega t / 2 Q_{0}}$. The main contribution to the distortion of the field in resonator is done by harmonics which are reflected with coefficients $/ \rho /=1$. The incident and reflected waves give standing wave:

$$
e^{i h_{1} z}+e^{-h_{s} z}=2 \cos \left(h_{s} z\right),
$$

which means that the distribution is described by $\cos \frac{\pi s}{N} n$, where $\mathrm{N}$ is the total number of cells in module and $n$ is number of cell. One can say that the integral over module of the total distortion (except the fundamental mode) equals zero. However, the front of the wave can have significant impact. For the simplest case, when we use linear dispersion, $\omega_{3}-\omega=\frac{\pi}{2} \frac{\omega K_{1}}{N}$, it is possible to get an analytical expression for the height of the wave [2]:

$$
\delta E_{1}=2 E_{1} R P \frac{L_{\text {cav }}}{v_{\text {group }}} \frac{\omega}{2 Q_{0}} e^{-\omega t / 2 Q_{0}}
$$

or using $P_{b e a m}=\frac{1}{2} I_{1} E_{1} \cos \phi_{s}$, one gets the expression by taking $R_{\text {shunt }}=E_{1}^{x} L_{\text {cavity }} / P_{\text {losses }}$ :

$$
\delta E_{1}=I_{1} R_{s h u n t} \frac{\tau_{g r}}{\tau_{t}} e^{-t / \tau_{t}},
$$

where $\tau_{g r}=L_{\text {cav }} / v_{g r}$ and $\tau_{t}=2 Q_{0} / \omega$. The value $K_{\tau}=\frac{\tau_{g r}}{\tau_{t}}$ defines the ratio between the front of the wave at the initial time and the final magnitude of the electromagnetic field obtained when we excite the resonator by a generator with the vector $I_{1} R_{s h u n t}$. For SSC case $K_{\tau}=0.35$. This means inputting a generator power into resonator with a shunt impedance $R_{\text {shunt }}=50 \mathrm{M} \Omega$ to compensate the beam loading with first a harmonic $I_{1}=2 I_{0}=2 * 0.02 \mathrm{~mA}$, then a wave is excited with a front amplitude equals to $0.7 \mathrm{Mv} / \mathrm{m}$. This is $10 \%$ of the accelerating harmonic.

\section{BEAM DYNAMICS}

Thus, during the transient the beam sees almost rectangular wave which radiates from the point of power input and damps simultaneously. In this section we shall do the analyze of the beam dynamics in this perturbed field.

The CCL structure has some features and one of them is a constant cell size in each tank which implies a constant phase velocity along the tank. So the equations for longitudinal motion are:

$$
\frac{d p}{d \psi}=\frac{e E_{p}}{2 \omega} \sin \phi
$$




$$
\frac{d \phi}{d \psi}=1-\frac{\beta_{, p}}{\beta},
$$

where $E_{p}$ is field which the particle sees, $p$ is the momentum of the particle, $\beta$ and $\phi$ are the relative velocity and phase of particle, $\beta_{w}$ is the phase velocity of the wave and $\psi$ is the normalized longitudinal coordinate which connects the longitudinal coordinate $z$ as $\psi=z / l_{c e l l}, l_{c e l l}$ is length of cell.

This system of equations can be represented by the second order, nonlinear, inhomogeneous, differentia equation:

$$
\begin{array}{r}
\frac{d^{2} \phi}{d \psi^{2}}+\frac{d(\ln \beta)}{d \psi} \frac{d \phi}{d \psi}+\Omega_{0}^{2}\left(\sin \phi-\sin \phi_{s}\right)= \\
\Omega_{0}^{2} \sin \phi_{s} \frac{\delta E_{1}}{E_{1}} \operatorname{Re} \sum_{t}^{N} 2 B_{s}(z, t)+\sum_{k} 2 \frac{\delta B_{v}}{\beta_{w}} \cos \frac{2 \pi k}{T} \psi
\end{array}
$$

where $\Omega_{0}^{2}=\frac{e E_{\gamma} \lambda B_{y}}{m_{0} c^{2} 2 \pi \gamma^{3} \beta^{2}}$ is the synchronous frequency for particles near the origin of the separatrix, $T$ is the periodicity of the tanks, $\delta E_{1} / E_{1}$ is the perturbation amplitude of the electromagnetic field during the transient, $\delta \beta_{w} / \beta_{\text {s }}$ is the jump of stepwise function of the phase velocity from tank to tank. One can see that even in an ideal structure we have the inhomogeneous equation what means that in the structure with constant cell size, the quasi-synchronons particle oscillates along the structure. During the transient the synchronous frequency becomes a function of the longitudinal coordinate $\psi$. Using (10) we can write the expression for the variable synchronous frequency $\Omega$ :

$$
\Omega^{2}=\Omega_{0}^{2}\left[1+\operatorname{Re} \sum_{s}^{N} 2 B_{s}(z, t)\right]
$$

Each harmonic has a distribution along the resonator of $\cos \left(\frac{\pi}{N}-\frac{\pi}{2}\right) \pi$. Taking into account that we have parametric and external resonances simultaneously the motion becomes unstable in the case when

$$
\nu=\frac{2 \Omega}{m},
$$

where $\nu$ is the frequency of the perturbation and $m$ is the order of resonance. However, one should remember that $\Omega$ here is the average synchronous frequency which depends on amplitude of oscillation (nonlinear resonance). In other words, we have many resonant conditions. The effective side band of resonance is defined by the perturbation amplitude and the degree of nonlinearity. Figure 2 shows the behavior of the quasi-synchronous frequency along the CCL accelerator. The vertical lines show the possible resonance frequency. Oscillation of the frequency is due to the constant phase velocity of the tank. If particles go through a resonance losses will be observed. Figure 3 a shows the separatrix if we go through resonance. It is clear that this situation is unacceptable for the accelerator. To avoid this effect we need to increase periodicity of the electromagnetic field perturbation. One solution is to input power in two symmetrical points at $1 / 4$ and $3 / 4$ of the module

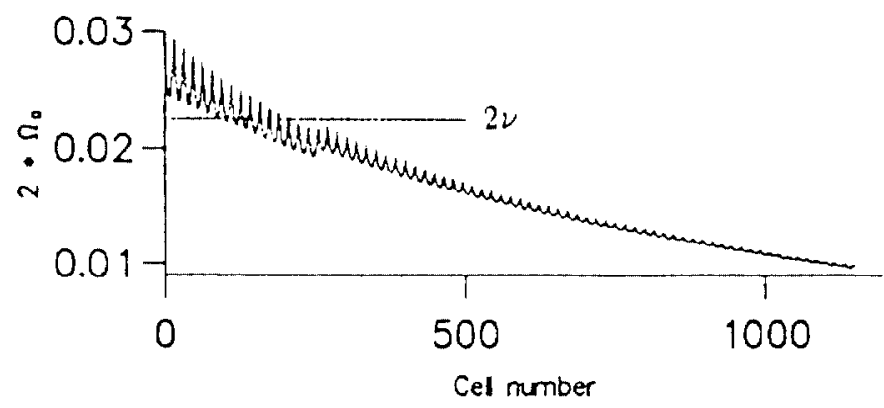

Figure 2. Synchronous frequency versus on cell namber

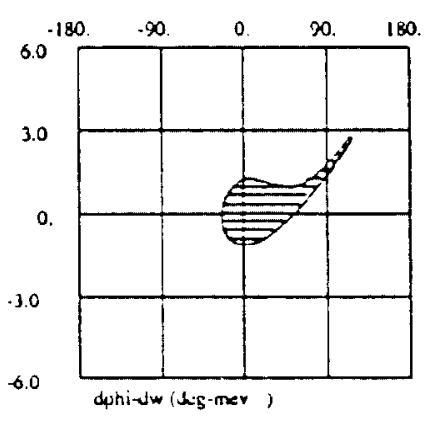

a.

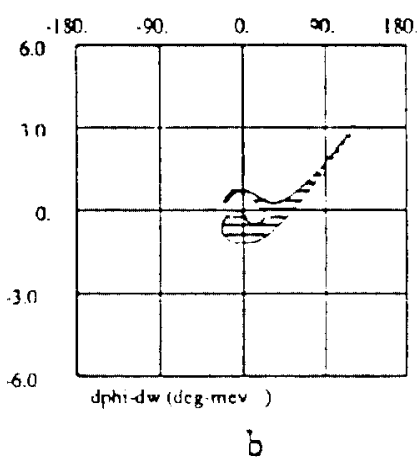

b
Figure 3. Corrected (a) and uncorrected (b) separatrix

length. Then the amplitude of the wave will decrease two times and the frequency of perturbation will increase two times giving the possibility of avoiding the resonant condition. On the same picture the case when we input the power in two points is shown (fig.3b). The separatrix has a quit perfect shape.

\section{CONCLUSION}

In conclusion we would like to make a general comment concerning accelerator design. Increasing the rate of acceleration increases the probability of resonance crossing. For the SSCL linac we pass through resonance in the second module. The maximum acceptable perturbation of electromagnetic field for our case is $2-3 \%$. To reduce the strength of a perturbing resonance, we have considered the method of resonance damping by "two points" power input.

In conclusion the authors would like to thank to Dr. W.Funk for his helpful discussion and support of this work.

\section{REFERENCES}

[1] L.Warren Funk, The SSC Linac, Linear Accelerator Proceedings,pp.8-12, 1992

[2] Yu.V.Senichev, Study of Injection Transient in Coupled Cell Linac Cavities, Third European Particle Accelerator Conference,pp.774-776, Berlin, 1992 